\title{
Anmerkungen zum Beitrag von Christian Holzner und Sonja Munz (2013): ,Should local public employment services be merged with local social benefit administrations?“ (Vol. 46, No. 2, S. 83-102)
}

\author{
Reinhard Hujer
}

Published online: 15 January 2014

(C) Institut für Arbeitsmarkt- und Berufsforschung 2014

Herr Prof. Dr. Joachim Wilde, Universität Osnabrück, hat zu diesem Artikel kritisch angemerkt, dass die für die empirische Analyse zentrale Variable ALP (für die Optionskommunen) keine statistische Signifikanz aufweist und somit die inhaltlichen Aussagen und politikrelevanten Folgerungen problematisch sind. Aus seiner Sicht besteht die Schlussfolgerung aus den publizierten Ergebnissen darin, dass sich keine statistisch gesicherten Unterschiede zwischen Optionskommunen und gemeinsamen Einrichtungen nachweisen lassen. Die Autoren haben sich mit dieser Kritik auseinandergesetzt. Sie haben zunächst darauf hingewiesen, dass sie unterschiedliche Gewichtungsansätze getestet haben, die die Signifikanzen beeinflusst haben. So hat eine Gewichtung mit der Zahl der Regionen ein signifikantes Ergebnis für die Optionskommunen ergeben. Bei Verwendung der Zahl der Arbeitslosengeld II-Bezieher wurde dagegen ein insignifikantes Ergebnis festgestellt. Unabhängig von den Gewichtungsalternativen zeigt sich in allen Spezifikationen die Tendenz, dass der Koeffizient für die Optionskommunen immer größer und negativ wird, wenn für organisatorische Merkmale der Institutionen kontrolliert wird. Der Unterschied zwischen den beiden Institutionsformen nimmt also zu. Dieses Ergebnis wird noch dadurch unterstützt, dass die Kreise der Optionskommunen vor der Reform eine signifikant niedrigere SozialhilfeEmpfängerquote hatten und somit eine effizientere Vermittlung zu erwarten wäre. Trotzdem bleibt festzuhalten, dass diese inhaltlich interessanten empirischen Ergebnisse der Studie als Tendenzaussagen $\mathrm{zu}$ interpretieren sind und weitere Analysen durchgeführt werden müssen, um politikrelevante Folgerungen auf der Grundlage signifikanter Schätzergebnisse ableiten zu können.

Diese Anmerkungen beziehen sich auf den Artikel, der unter doi:10.1007/s12651-012-0117-7 verfügbar ist.

\section{R. Hujer ( $\square)$}

Goethe-Universität, Frankfurt a.M., Deutschland

e-mail: reiner.hujer@gmx.de 